PSI-PR-96-19

\title{
A simple method for multi-leg loop calculations
}

\author{
R. Pittau* \\ Paul Scherrer Institute, CH-5232 Villigen-PSI, Switzerland
}

September 10, 2021

\begin{abstract}
In this paper, I present a technique to simplify the tensorial reduction of one-loop integrals with arbitrary internal masses, but at least two massless external legs. By applying the method to rank $l$ tensor integrals, one ends up with at most rank 1 tensor functions with the initial number of denominators, plus tensor integrals with less denominators and rank $<l$. To illustrate the algorithm, I explicitly compute diagrams contributing to processes of physical interest and show how the usual numerical instabilities due to the appearance of Gram determinants can be controlled.
\end{abstract}

*email address: pittau@psw218.psi.ch 


\section{Introduction}

Present and future experiments in high energy physics require an increasing understanding of multi-particle final states. Idealized calculations, in which heavy particles are treated as being on-shell, are often not accurate enough to match the experimental needs. In fact, the produced heavy particles always decay, giving a multi-body final state as an observable signal.

At first, investigations of multi-particle processes can be performed at the tree level, but, in a second stage, also radiative corrections have to be taken into account. At LEP2, for instance, a complete electroweak one-loop calculation to off-shell four-fermion production beyond the leading log approximation is still missing, and this reflects on the accuracy in the $W$ mass measurement. Besides, next-to-leading order QCD calculations of multi-jet final states are becoming unavoidable to control the background in the search of new signals. Therefore, it is clear that multi-leg loop calculations are playing a role of increasing importance and that any progress achieved in this field is welcome.

One of the major problems in loop calculations is the reduction (in $n$ dimensions) of rank $l$ tensor $m$-points integrals as follows

$$
\begin{aligned}
I_{m}^{\mu_{1} \cdots \mu_{l}} & =\int d^{n} q \frac{q^{\mu_{1}} \cdots q^{\mu_{l}}}{D_{1} \cdots D_{m}} \\
D_{i} & =\left(q+s_{i-1}\right)^{2}-m_{i}^{2} \\
s_{i} & =\sum_{j=0}^{i} k_{j} \quad k_{0}=0 .
\end{aligned}
$$

The standard reduction method [1] consists in decomposing the tensorial structure in terms of momenta $k_{j}$ and metric tensor. For example, a rank two 5-point one-loop integral can be expressed in terms of 11 linearly independent scalar form factors

$$
\begin{aligned}
I_{5}^{\mu \nu} & =\int d^{n} q \frac{q^{\mu} q^{\nu}}{D_{1} D_{2} D_{3} D_{4} D_{5}} \\
& =E_{21} k_{1}^{\mu} k_{1}^{\nu}+E_{22} k_{2}^{\mu} k_{2}^{\nu}+\cdots+E_{211} g^{\mu \nu} .
\end{aligned}
$$

Analogously, the rank three 5-point function $I_{5}^{\mu \nu \rho}$ gives 24 terms.

The freedom in choosing the basis for the tensorial decomposition can be used to define suitable combinations $v_{j}$ of $k_{j}$ such that $v_{i} \cdot k_{j}=\delta_{i j}$ [2]. In 
any case, in order to express the coefficients of the decomposition in terms of scalar loop functions, one has to solve algebraical systems obtained by multiplying both sides of eq. (11) by $k_{j}$ (or linear combinations of them) and the metric tensor. This causes the appearance of Gram determinants

$$
\Delta=\operatorname{det}\left(k_{i} \cdot k_{j}\right)
$$

into the denominator, that can give rise to numerical instabilities. In fact, they vanish in collinear regions of the phase space where the cross section is well defined, so that large numerical cancellations are expected in the numerator in order to keep everything finite.

Different approaches also exist in which the form factors are identified with integrals in a different number of dimensions [3] or directly formulated in the Feynman parameter space [4]. In both cases, Gram determinants still appear at some stage in the reduction.

Recently, a new method has been proposed by Campbell, Glover and Miller [5], in which the coefficients of the reduction are built up by combining the scalar integrals in groupings that are well behaved in the limit $\Delta \rightarrow 0$. This somehow solves the problem of the Gram determinants, but to actually construct the well behaved groupings can be cumbersome in general - non QCD like - cases, especially when the rank of the appearing tensor integrals is large. Furthermore, the number of the basic functions increases, and additional scalar integrals must be evaluated in $n=6+\epsilon$ or higher dimensions.

In this paper, I propose a method to simplify Feynman diagrams, in which no evaluation of new functions is required, apart from the usual scalar integrals (new functions may appear when $n=4+\epsilon$, but their computation is trivial). Although, in general, the appearance in the denominator of quantities that can vanish in some corner of the phase space cannot be completely avoided, a better control of those singularities is possible with respect to the traditional methods of refs. [1, 2]. The procedure is somehow complementary to the technique of Campbell, Glover and Miller, and can be used to simplify the problem before applying their method.

A trivial example serves to illustrate the basic idea. Consider the tensorial reduction of the following quantity

$$
\gamma_{\mu} \gamma_{\nu} I_{5}^{\mu \nu}=\int d^{n} q \frac{\not q \not 1}{D_{1} D_{2} D_{3} D_{4} D_{5}} .
$$


One can certainly use eq. (2), but the equation

$$
\not 1 \not 1=q^{2}=D_{1}+m_{1}^{2}
$$

immediately gives the desired answer in terms of just two scalar functions

$$
\gamma_{\mu} \gamma_{\nu} I^{\mu \nu}=\int d^{n} q \frac{1}{D_{2} D_{3} D_{4} D_{5}}+m_{1}^{2} \int d^{n} q \frac{1}{D_{1} D_{2} D_{3} D_{4} D_{5}} .
$$

Therefore, by using in a diagram the algebra of the $\gamma$ matrices to reconstruct denominators, one gets a simpler tensorial structure.

This technique of reconstructing denominators has been already used in the literature for the evaluation of specific integrals [6, 7]. In this paper, I show that a reduction based on this procedure can always be worked out for generic one-loop integrals with arbitrary internal masses, but at least two massless external legs.

In the next section, I present the method, with the help two 4-dimensional examples. In section 3 and appendix A, I describe the extension to $n=4+\epsilon$ dimensions and give explicit results for cases of physical interest.

\section{The Method}

After the decay of the intermediate heavy particles, a generic $m$-point loop diagram can be written as follows (see fig. 1-a)

$$
\mathcal{M}\left(p_{1}, \cdots, p_{r} ; k_{1}, \cdots, k_{m-1}\right)=\sum_{i}^{N} \int d^{n} q \frac{\operatorname{Tr}^{(i)}[\not 1 \cdots \not 1 \cdots]}{D_{1} \cdots D_{m}} \equiv \sum_{i}^{N} \mathcal{M}^{(i)},
$$

where $p_{1 \cdots r}$ are the $r$ external momenta of the diagram, $k_{1 \cdots m-1}$ the momenta in the loop functions - as defined in eq. (11) - and $\operatorname{Tr}^{(i)}$ traces over $\gamma$ matrices, which contain $\not$ 's. In most practical cases, the $r$ external momenta are massless, in fact they can only be photons, gluons or light fermions. The appearance of traces in eq. (5) is a general feature. In fact, if the external particles of the diagram are fermions, traces naturally appear, while the polarization vectors of photons and gluons can always be written in terms of spinor strings using the following representation [8]

$$
\epsilon^{\mu}(\lambda, k)=\frac{1}{\sqrt{2}} \frac{\bar{v}_{-\lambda}(k) \gamma^{\mu} u_{\lambda}(b)}{\bar{v}_{\lambda}(b) u_{\lambda}(k)} \quad b=\text { arbitrary massless vector } .
$$


In standard calculations, each term in eq. (5) is rewritten as follows

$$
\mathcal{M}^{(i)}\left(p_{1}, \cdots, p_{r} ; k_{1}, \cdots, k_{m-1}\right)=\int d^{n} q \frac{q^{\alpha} \cdots q^{\beta} \cdots \operatorname{Tr}^{(i)}\left[\gamma_{\alpha} \cdots \gamma_{\beta} \cdots\right]}{D_{1} \cdots D_{m}}
$$

and the tensors

$$
\int d^{n} q \frac{q^{\alpha} \cdots q^{\beta} \cdots}{D_{1} \cdots D_{m}}
$$

decomposed using a basis of independent four-vectors, as described in the introduction. Instead, I propose to use $\gamma$-algebra and spinor manipulations to move all $\not$ 's close to each other, and subsequently apply $\not \phi \phi=q^{2}$ in order to rewrite each trace as

$$
\operatorname{Tr}^{(i)}[\not \not \cdots \not l \cdots]=\sum_{k} A_{k} \operatorname{Tr}_{k}^{(i)}[\not 1 \cdots]+\sum_{l} B_{l} \operatorname{Tr}_{l}^{(i)}[\cdots]
$$

where $\operatorname{Tr}_{k}^{(i)}$ contain at most one $\not, \operatorname{Tr}_{l}^{(i)}$ do not contain $\not$ and the coefficients $A_{k}$ and $B_{l}$ are functions of the scalar products between $q$ and the external momenta of the diagram $p_{1 \cdots r}$. Since a single $\not$ appears in the right side of eq. (9), just a rank 1 tensor decomposition is formally left. Anyway, there is no gain in applying the described procedure if the coefficients $A_{k}$ and $B_{l}$ are generic. In fact, if scalar products of the kind $\left(q \cdot p_{j}\right)$ appear and $p_{j}$ is not equal to one of the momenta $k_{j}$ in the loop functions, the same kind of tensorial decomposition necessary for computing eq. (8) is hidden in eq. (9). On the other hand, if only powers of $\left(q \cdot k_{j}\right)$ and $q^{2}$ appear, it is always possible to express at least one of them in terms of denominators. In this case, starting from $m$-point rank $l$ tensor integrals, the algorithm gives at most rank $1 \mathrm{~m}$-point functions, plus $n$-point rank $p$ tensor integrals with $n<m$ and $p<l$.

Naturally the question arises whether the coefficients $A_{k}$ and $B_{l}$ can be made to have such a nice feature. To answer that question, while keeping everything transparent, I will assume, for the rest of this section, four-dimensional space time, namely ultraviolet finite integrals, with four-dimensional regularization of infrared and collinear divergences. The extension to $n=4+\epsilon$ dimensions will be considered in the next section. In the following, I introduce all notations and necessary formulae. 
Basic objects are strings of $\gamma$ matrices between massless spinors. I will use the notation

$$
\bar{v}_{+}(i) \not p_{3} \not p_{4} \not \not p_{5} \cdots \not p_{6} u_{-}(j)=\{34 q 5 \cdots 6\}_{i j}^{+-} .
$$

The Weyl spinors are defined as

$$
\begin{array}{ll}
u_{ \pm}(j)=\Pi_{ \pm} u(j) & \bar{u}_{ \pm}(j)=\bar{u} \Pi_{\mp} \\
v_{ \pm}(j)=\Pi_{\mp} v(j) & \bar{v}_{ \pm}(j)=\bar{v} \Pi_{ \pm},
\end{array}
$$

where $j$ denotes the spinor momentum and $\Pi_{ \pm}=\frac{1}{2}\left(1 \pm \gamma_{5}\right)$ are chirality projectors obeying

$$
\Pi_{+} \Pi_{-}=0 \quad\left(\Pi_{ \pm}\right)^{2}=\Pi_{ \pm} .
$$

Note that, using $u_{ \pm}(j)=v_{\mp}(j)$, one can always rewrite any spinorial string with $v$ spinors on the left and $u$ spinors on the right, so that the notation in eq. (10) is completely general.

The completeness relations for massless spinors read

$$
\begin{aligned}
& u_{ \pm}(j) \bar{u}_{ \pm}(j)=\not \Pi_{j} \Pi_{\mp} \\
& v_{ \pm}(j) \bar{v}_{ \pm}(j)=\not \prod_{j} \Pi_{ \pm},
\end{aligned}
$$

from which one can easily get similar relations for generic momenta $i$ and $j$ [9]

$$
\begin{aligned}
u_{-}(i) \bar{v}_{-}(j) & =-\frac{\not p_{i} p_{j}}{<i j>} \Pi_{-} \\
u_{+}(i) \bar{v}_{+}(j) & =\frac{p_{i} p_{j}}{<i j>^{*}} \Pi_{+} \\
u_{-}(i) \bar{v}_{+}(j) & =\frac{\not p_{i} \not p_{j}}{\bar{v}_{+}(i) \not u_{-}(j)} \Pi_{+} \\
u_{+}(i) \bar{v}_{-}(j) & =\frac{\not p_{i} \phi p_{j}}{\bar{v}_{-}(i) \not u_{+}(j)} \Pi_{-},
\end{aligned}
$$

where $b$ is an arbitrary four-vector and

$$
\begin{aligned}
& \langle i j\rangle^{*}=\bar{v}_{-}(i) u_{-}(j)=\bar{u}_{+}(i) v_{+}(j) \\
& <i j\rangle=-\bar{v}_{+}(i) u_{+}(j)=-\bar{u}_{-}(i) v_{-}(j)
\end{aligned}
$$


are scalar products in the spinor space [8, 10].

The following identities hold for spinor strings [11]

$$
\begin{aligned}
\{\Gamma\}_{i j}^{+-} & =\{\tilde{\Gamma}\}_{j i}^{-+} \\
\{\Gamma\}_{i j}^{ \pm \pm} & =-\{\tilde{\Gamma}\}_{j i}^{ \pm \pm},
\end{aligned}
$$

$\tilde{\Gamma}$ being the string obtained from $\Gamma$ by reversing the order of the $\gamma$ matrices. The following Kahane-Chisholm identities [12] are also very useful

$$
\gamma_{\alpha} \Gamma_{o} \gamma^{\alpha}=-2 \tilde{\Gamma}_{o} \quad \gamma_{\alpha} \Gamma_{e} \gamma^{\alpha}=\operatorname{Tr}\left[\Gamma_{e}\right]-\gamma_{5} \operatorname{Tr}\left[\gamma_{5} \Gamma_{e}\right],
$$

where $\Gamma_{o}$ and $\Gamma_{e}$ stand for strings with an odd and even number of $\gamma$ matrices, respectively. Finally, one can prove [1]

$$
\not \Gamma_{e} \not b=-\not \tilde{\Gamma}_{e} \not \quad \text { if } \quad b^{2}=0 .
$$

Armed with the previous formulae, I will show that, in four dimensions, the coefficients $A_{k}$ and $B_{l}$ in eq. (9) can be made to depend only on powers of $q^{2}$ and $\left(q \cdot k_{j}\right)$ on the condition that at least two momenta in the set $k_{1 \cdots m-1}$ (say $k_{1}$ and $k_{2}$ ) are massless, namely coincide with some of the external massless momenta of the diagram, as in fig. 1-b and 1-c.

In fact, the following identity

$$
\not q=\frac{1}{2\left(k_{1} \cdot k_{2}\right)}\left[2\left(q \cdot k_{2}\right) \not k_{1}+2\left(q \cdot k_{1}\right) \not k_{2}-\not k_{1} \phi k_{2}-\not k_{2} \phi \not k_{1}\right]
$$
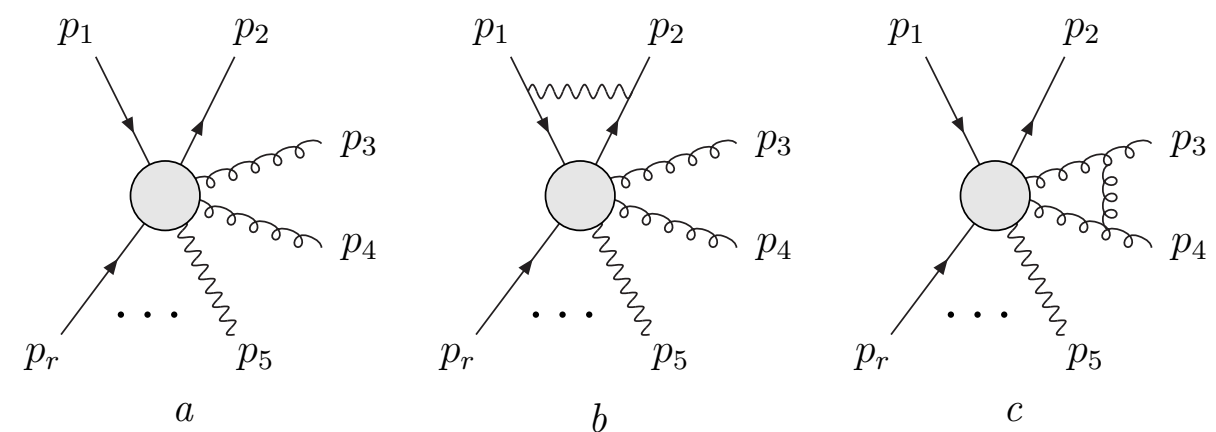

Figure 1: Generic one-loop multi-leg process (a). The blob stands for the sum of all possible one-loop diagrams. Two classes of diagrams are also shown, for which the reduction method is applicable ( $b$ and $c$ ). 
allows to "extract" $q$ from the traces

$$
\begin{aligned}
\operatorname{Tr}\left[\Gamma_{1} \not \Gamma_{2}\right] & =\frac{1}{2\left(k_{1} \cdot k_{2}\right)}\left\{2\left(q \cdot k_{2}\right) \operatorname{Tr}\left[\Gamma_{1} \not k_{1} \Gamma_{2}\right]+2\left(q \cdot k_{1}\right) \operatorname{Tr}\left[\Gamma_{1} \not k_{2} \Gamma_{2}\right]\right. \\
& \left.-\operatorname{Tr}\left[\Gamma_{1} \not k_{1} \phi k_{2} \Gamma_{2}\right]-\operatorname{Tr}\left[\Gamma_{1} \not k_{2} \not \not k_{1} \Gamma_{2}\right]\right\},
\end{aligned}
$$

where $\Gamma_{1,2}$ represent generic strings of $\gamma$ matrices. In the first two terms, the $q$ dependence is already extracted out. The other two traces can be broken with the help of eq. (13)

$$
\begin{aligned}
\operatorname{Tr}\left[\Gamma_{1} \not k_{1} \phi \not k_{2} \Gamma_{2}\right] & =\operatorname{Tr}\left[\Gamma_{1} \not k_{1} \phi k_{2}\left(\Pi_{+}+\Pi_{-}\right) \Gamma_{2}\right] \\
& =\{q\}_{12}^{+-}\left\{\Gamma_{2} \Gamma_{1}\right\}_{21}^{+-}+\{q\}_{12}^{-+}\left\{\Gamma_{2} \Gamma_{1}\right\}_{21}^{-+} .
\end{aligned}
$$

By iteratively applying the above procedure together with the identities (derived again by means of eq. (13) and eq. (14))

$$
\begin{aligned}
\{q\}_{12}^{-+}\{q\}_{21}^{-+} & =4\left(q \cdot k_{1}\right)\left(q \cdot k_{2}\right)-2 q^{2}\left(k_{1} \cdot k_{2}\right) \\
\{q\}_{12}^{-+}\{q\}_{12}^{-+} & =\frac{1}{\bar{v}_{+}(1) \not u_{-}(2)}\left[2\left(q \cdot k_{1}\right)\{b 2 q\}_{21}^{+-}-2(q \cdot b)\{12 q\}_{21}^{+-}\right. \\
& \left.+2\left(q \cdot k_{2}\right)\{1 b q\}_{21}^{+-}-q^{2}\{1 b 2\}_{21}^{+-}\right]
\end{aligned}
$$

it is easy to see that the resulting coefficients $A_{k}$ and $B_{l}$ only depend on powers of $q^{2},\left(q \cdot k_{1}\right),\left(q \cdot k_{2}\right)$ and $(q \cdot b)$. The vector $b$ is arbitrary (and not necessarily massless), but different from $k_{1}$ and $k_{2}$. By identifying it with a third momentum in the set $k_{1 \cdots m-1}$ 凹, $A_{k}$ and $B_{l}$ have the suitable structure to get the desired simplifications in the tensorial decomposition.

In the computation of physical processes, the one-loop diagrams with highest $m$ always lie in the class of the corrections connecting two external legs (see again fig. 1-b and 1-c). That guarantees at least two massless momenta in the loop functions and, therefore, that the described reduction method is applicable at least for the most complicated diagrams appearing in the calculation.

Finally, one should notice that structures that can vanish appear in the denominator of eqs. (20) and (22). However, as we will see, often in practical

\footnotetext{
${ }^{1}$ This identification is possible only for $m$-point loop functions with $m>3$. However, in the next section, I will show that the method still works when $m \leq 3$.
} 
calculations there is no need to extract the $q$ dependence as described, since the reconstruction of denominators naturally takes place without introducing too many factors in the denominators.

In the following two examples, I show the method at work in four dimensions.

\section{Example 1}

I shall compute, in the renormalizable gauge, the 6-point diagram of fig. 2, relevant for studying electroweak corrections at LEP2.

The spinorial structure in the numerator of the diagram reads

$$
\begin{aligned}
\mathcal{A} & =-\bar{v}_{+}(3) \gamma_{\mu} p_{B} \gamma_{\beta} u_{-}(4) \bar{v}_{+}(5) \gamma^{\beta} \not p_{A} \gamma^{\nu} u_{-}(6) \bar{v}_{+}(1) \gamma^{\mu} p_{C} \gamma_{\nu} u_{-}(2) \\
& \equiv-\{\mu B \beta\}_{34}^{+-}\{\beta A \nu\}_{56}^{+-}\{\mu C \nu\}_{12}^{+-}
\end{aligned}
$$

from which the scattering amplitude $\mathcal{M}$ can be obtained after division by the denominators and integration over $d^{4} q$

$$
\mathcal{M}=\int d^{4} q \frac{\mathcal{A}}{D_{1} D_{2} D_{3} D_{4} D_{5} D_{6}}
$$

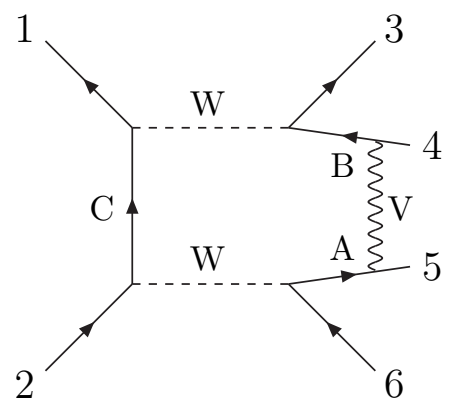

$$
\begin{aligned}
& p_{A}=q \quad p_{B}=q+p_{4}+p_{5} \\
& p_{C}=q-p_{2}-p_{6} \\
& D_{1}=q^{2} \\
& D_{2}=\left(q+p_{5}\right)^{2}-M_{V}^{2} \\
& D_{3}=\left(q+p_{5}+p_{4}\right)^{2} \\
& D_{4}=\left(q+p_{5}+p_{4}+p_{3}\right)^{2}-M_{W}^{2} \\
& D_{5}=\left(q+p_{5}+p_{4}+p_{3}+p_{1}\right)^{2} \\
& D_{6}=\left(q+p_{5}+p_{4}+p_{3}+p_{1}+p_{2}\right)^{2}-M_{W}^{2}
\end{aligned}
$$

Figure 2: LEP2 6-point diagram. All momenta are incoming. $V$ can be either a massive gauge boson or a photon. In the latter case, an appropriate four-dimensional infrared regularization is understood.

Using eq. (14) one can rewrite

$$
\mathcal{A}=-\frac{1}{N}\{\mu B \beta 4 b 5 \beta A \nu 6 b 1 \mu C \nu\}_{32}^{+-}
$$




$$
\begin{aligned}
& =-\frac{4}{N}\{1 b 6 \nu A 4 b 5 B C \nu\}_{32}^{+-} \\
N & =\bar{v}_{+}(4) \not b u_{-}(5) \bar{v}_{+}(6) \not b u_{-}(1),
\end{aligned}
$$

where I have applied twice the Chisholm identity in eq. (17) to sum over $\beta$ and $\mu$. By introducing a new summed four-dimensional index $\alpha$, the products $u_{-}(i) \bar{v}_{+}(j)$ can be reconstructed

$$
\mathcal{A}=2 \bar{v}_{+}(3) \gamma_{\alpha} u_{-}(6) \bar{v}_{+}(1) \gamma^{\alpha} \gamma^{\nu} \not p_{A} u_{-}(4) \bar{v}_{+}(5) \not p_{B} \not p_{C} \gamma_{\nu} u_{-}(2)
$$

and, after Fierz reordering the last two strings by means of the second of eqs. (17), one gets

$$
\mathcal{A}=4 \bar{v}_{+}(3) \gamma_{\alpha} u_{-}(6) \bar{v}_{+}(1) \gamma^{\alpha} u_{-}(2) \bar{v}_{+}(5) \not p_{B} p_{C} p_{A} u_{-}(4) \text {. }
$$

Since

$$
\begin{aligned}
\not p_{B} \not p_{C} \not p_{A} & =\left[D_{5}+\not p_{13} \not p_{26}\right] \not q-D_{1} \not p_{13} \\
p_{i j} & =\not p_{i}+\not p_{j}
\end{aligned}
$$

denominators naturally appear in $\mathcal{A}$, so that the scattering amplitude is immediately expressed in terms of one scalar 5-point function, one rank one 5 -point function and one 6-point function or rank 1.

\section{Example 2}

A different contribution comes from the "crossed" 6-point diagram in fig. 3 .

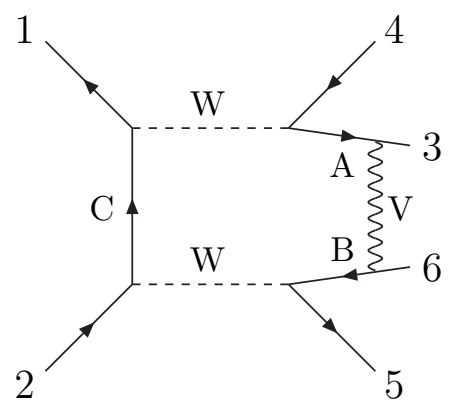

$$
\begin{aligned}
& p_{A}=q \quad p_{B}=q+p_{3}+p_{6} \\
& p_{C}=q-p_{1}-p_{4} \\
& D_{1}=q^{2} \\
& D_{2}=\left(q+p_{3}\right)^{2}-M_{V}^{2} \\
& D_{3}=\left(q+p_{3}+p_{6}\right)^{2} \\
& D_{4}=\left(q+p_{3}+p_{6}+p_{5}\right)^{2}-M_{W}^{2} \\
& D_{5}=\left(q+p_{3}+p_{6}+p_{5}+p_{2}\right)^{2} \\
& D_{6}=\left(q+p_{3}+p_{6}+p_{5}+p_{2}+p_{1}\right)^{2}-M_{W}^{2}
\end{aligned}
$$

Figure 3: "Crossed" LEP2 6-point diagram. 
The numerator of the amplitude reads

$$
\mathcal{A}=\{\mu C \nu\}_{12}^{+-}\{\nu B \beta\}_{56}^{+-}\{\beta A \mu\}_{34}^{+-} .
$$

Exactly as before, one easily performs the sums over $\mu, \nu$ and $\beta$

$$
\begin{array}{r}
\mathcal{A}=-8\{A\}_{16}^{+-}\{B\}_{32}^{+-}\{C\}_{54}^{+-}=8\left[\{q\}_{16}^{+-}\{6\}_{32}^{+-}\{1\}_{54}^{+-}\right. \\
\left.-\{q\}_{16}^{+-}\{6\}_{32}^{+-}\{q\}_{54}^{+-}+\{q\}_{16}^{+-}\{q\}_{32}^{+-}\{1\}_{54}^{+-}-\{q\}_{16}^{+-}\{q\}_{32}^{+-}\{q\}_{54}^{+-}\right] .
\end{array}
$$

The first term in eq. (29) only contains one $q$ and cannot be further manipulated, while the second and the third term can be reduced with the help of eq. (14). For example

$$
\begin{aligned}
& \{q\}_{16}^{+-}\{q\}_{54}^{+-}=\{q\}_{16}^{+-}\{q\}_{45}^{-+}=-\frac{1}{<64>}\{q 64 q\}_{15}^{++} \\
= & -\frac{1}{<64>}\left[2\left(q \cdot p_{6}\right)\{4 q\}_{15}^{++}-2\left(q \cdot p_{4}\right)\{6 q\}_{15}^{++}+q^{2}\{64\}_{15}^{++}\right] .
\end{aligned}
$$

Similarly, one gets for the last term

$$
\begin{gathered}
\{q\}_{16}^{+-}\{q\}_{32}^{+-}\{q\}_{54}^{+-}=\{q\}_{16}^{+-}\{q\}_{23}^{-+}\{q\}_{54}^{+-} \\
=-\frac{1}{<62><35>^{*}}\{q 62 q 35 q\}_{14}^{+-}= \\
-\frac{1}{<62><35>^{*}}\left[4\left(q \cdot p_{6}\right)\left(q \cdot p_{3}\right)\{25 q\}_{14}^{+-}-4\left(q \cdot p_{6}\right)\left(q \cdot p_{5}\right)\{23 q\}_{14}^{+-}\right. \\
+2\left(q \cdot p_{6}\right) q^{2}\{235\}_{14}^{+-}-4\left(q \cdot p_{2}\right)\left(q \cdot p_{3}\right)\{65 q\}_{14}^{+-} \\
\left.+4\left(q \cdot p_{2}\right)\left(q \cdot p_{5}\right)\{63 q\}_{14}^{+-}-2\left(q \cdot p_{2}\right) q^{2}\{635\}_{14}^{+-}+q^{2}\{6235 q\}_{14}^{+-}\right] \cdot(31)
\end{gathered}
$$

Therefore, by reconstructing denominators from the dot products, one easily see that the most complicated objects appearing in the scattering amplitude are rank one 6-point functions and rank two 5-point functions, as expected.

Some comments are in order. What I have done in the previous examples is equivalent to Fierz reordering the spinorial strings. This already gives the correct structure to apply the reduction algorithm, without using eq. (19). The fact that some diagrams are simpler to compute than others, depends on what one gets after Fierz reordering. In fact, after elimination of all sum 
indexes, one can end up with momenta containing $q$ (that I denote with capital letters) either close to each other or "sandwiched" between spinors. Namely

$$
\{A B C \cdots\}_{i j}^{+-}
$$

or

$$
\{A\}_{i j}^{+-}\{B\}_{k l}^{+-}\{C\}_{m n}^{+-} \cdots \text {. }
$$

In the first case, the final result is simpler because the equation $\not \phi q=q^{2}$ can be immediately applied, while, in the second case, one first needs to use eqs. (14) and some $\gamma$-algebra.

In example 1 there are no singular structures, while, in example 2 , a single quantity, that can vanish in the collinear limit, appears in the denominator (see eqs. (30) and (31)). This roughly corresponds to a situation with a single Gram determinant in the denominator, that is known to be numerically quite stable [5] (actually, since $<i j><i j>^{*}=2\left(p_{i} \cdot p_{j}\right)$ eqs. (30) and (31) are even more stable). In addition, even in this second case, one can in principle get rid of all singularities by changing the reduction when the collinear limit

is approached. For example, a reduction equivalent to eq. (31), but with a different singular structure, can be obtained by replacing

$$
\begin{array}{lll}
1 \rightarrow 3 & 2 \rightarrow 4 & 3 \rightarrow 5 \\
4 \rightarrow 6 & 5 \rightarrow 1 & 6 \rightarrow 2
\end{array}
$$

In conclusion, one has a better control on the singularities, with respect to traditional reduction methods, in which one would have immediately to start with rank three 6-point functions and a higher number of Gram determinants.

\section{The $n$-dimensional case}

In this section, I show how the method can be applied in $n$ dimensions.

The basic idea is simple. Starting from the traces in eq. (5) one breaks each $n$-dimensional vector into four-dimensional and $\epsilon$-dimensional part [6, 13, 14] $(\epsilon=n-4)$. This allows to factorize any $\epsilon$ dependence out of four-dimensional objects, in which all properties of the spinor calculus can still be used in order 
to work out the reduction, exactly as shown in the previous section. This procedure is equivalent to assume a four-dimensional-helicity regularization scheme, in which only the loop momentum is continued in $4+\epsilon$ dimensions, while helicity states are kept four-dimensional [15].

Before illustrating the procedure with explicit examples, I introduce notations and relevant formulae.

I underline quantities that live in the $n$-dimensional space, while a tilde denotes $\epsilon$-dimensional objects. A generic $n$-dimensional vector $\underline{v}$ can then be written as

$$
\underline{v}=v+\tilde{v} .
$$

It is always possible to chose orthogonal spaces for 4 and $\epsilon$-vectors, such that $v_{i} \cdot \tilde{v}_{j}=0$. Besides, $\epsilon$-dimensional $\gamma$ matrices freely anticommute with four-dimensional ones

$$
\left\{\gamma_{\mu}, \tilde{\gamma}_{\nu}\right\}=0
$$

As for $\gamma_{5}$, a mathematical correct prescription would be keeping its definition also in $n-4$ dimensions, that implies

$$
\left[\gamma_{5}, \tilde{\gamma}_{\mu}\right]=0
$$

While this causes no trouble for diagrams with only infrared or collinear divergences, extra finite terms, that violate the Ward identities, appear when ultraviolet divergences are present. At the one-loop level, one may either adopt eq. (35) and restore the Ward identities, by introducing appropriate finite counter terms in the Lagrangian, or impose, also for $\epsilon$-dimensional matrices

$$
\left\{\gamma_{5}, \tilde{\gamma}_{\mu}\right\}=0
$$

plus extra care in manipulating traces [16]. In the following, I always assume eq. (36). To preserve consistency, it is enough anticommuting $\gamma_{5}$ to the end of a trace before performing any $\gamma$-algebra [17.

As a first example, I compute the simple vertex diagram in fig. 4. 


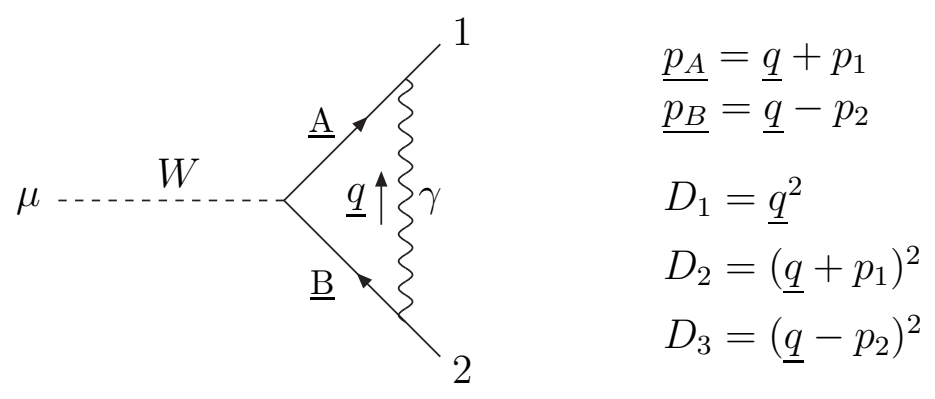

Figure 4: Vertex diagram in $n$ dimensions.

Splitting up the $n$-dimensional traces as in ref. [14], the numerator of the amplitude can be written as follows

$$
\begin{aligned}
\mathcal{A}^{\mu} & =\left\{\underline{\beta} \underline{A} \mu \Pi_{-} \underline{B} \underline{\beta}\right\}_{12}^{+-}=\{\underline{\beta} \underline{A} \mu \underline{B} \underline{\beta}\}_{12}^{+-} \\
& =\{\beta A \mu B \beta\}_{12}^{+-}-\epsilon\{A \mu B\}_{12}^{+-}-\tilde{q}^{2}\{\beta \mu \beta\}_{12}^{+-}+\epsilon \tilde{q}^{2}\{\mu\}_{12}^{+-},
\end{aligned}
$$

where all $\epsilon$-dimensional $\gamma$-algebra has been explicitly worked out in order to get the desired factorization of the $\epsilon$-dimensional objects. Summing over $\beta$ gives

$$
\mathcal{A}^{\mu}=-2\{B \mu A\}_{12}^{+-}-\epsilon\{q \mu q\}_{12}^{+-}+\tilde{q}^{2}(2+\epsilon)\{\mu\}_{12}^{+-},
$$

where the Dirac equation has also been used.

In the first term of eq. (38), $A$ and $B$ can be extracted with the help of eq. (20)

$$
\begin{gathered}
\{B \mu A\}_{12}^{+-}=\frac{1}{4\left(p_{1} \cdot p_{2}\right)^{2}}\left[4\left(p_{A} \cdot p_{2}\right)\left(p_{B} \cdot p_{1}\right)\{2 \mu 1\}_{12}^{+-}\right. \\
\left.-2\left(p_{B} \cdot p_{1}\right)\{2 \mu 2 q 1\}_{12}^{+-}-2\left(p_{A} \cdot p_{2}\right)\{2 q 1 \mu 1\}_{12}^{+-}+\{2 q 1 \mu 2 q 1\}_{12}^{+-}\right] .
\end{gathered}
$$

One easily convinces oneself that the last three terms of eq. (39) do not contribute to the amplitude. In fact, performing a four-dimensional tensorial decomposition, each coefficient gives zero. Therefore, one gets the following result in terms of denominators

$$
\{B \mu A\}_{12}^{+-}=\{\mu\}_{12}^{+-}\left[\frac{D_{1}-D_{2}}{p_{1} \cdot p_{2}}\left(q \cdot p_{2}\right)+2 D_{1}-D_{2}-D_{3}+2\left(p_{1} \cdot p_{2}\right)\right] \text {. }
$$


Analogously

$$
\{q \mu q\}_{12}^{+-}=\{\mu\}_{12}^{+-} \frac{D_{1}-D_{2}}{p_{1} \cdot p_{2}}\left(q \cdot p_{2}\right)
$$

and the final result reads

$$
\begin{aligned}
\mathcal{A}^{\mu} & =\{\mu\}_{12}^{+-}\left[(2+\epsilon)\left(\tilde{q}^{2}+\frac{D_{2}-D_{1}}{p_{1} \cdot p_{2}}\left(q \cdot p_{2}\right)\right)\right. \\
& \left.-4\left(p_{1} \cdot p_{2}\right)+2\left(D_{3}+D_{2}-2 D_{1}\right)\right] .
\end{aligned}
$$

In the previous equation, the desired reconstruction of denominators has been achieved, but a term proportional to $\tilde{q}^{2}$ is also left, that requires the evaluation of the extra scalar integral

$$
\tilde{I}_{3}=\int d^{n} q \frac{\tilde{q}^{2}}{D_{1} D_{2} D_{3}} .
$$

By decomposing the integration as follows 18

$$
\int d^{n} q=\int d^{4} q d^{\epsilon} \mu \quad \text { with } \quad \tilde{q}^{2}=-\mu^{2}
$$

one easily gets

$$
\tilde{I}_{3}=-i \frac{\pi^{2}}{2}+\mathcal{O}(\epsilon)
$$

that is all one needs.

The result in eq. (42) can be checked by using standard reduction techniques.

As a second example, I compute the box diagram in fig. 5, contributing to the one-loop QCD corrections to 4 fermion production at LEP2 [19].

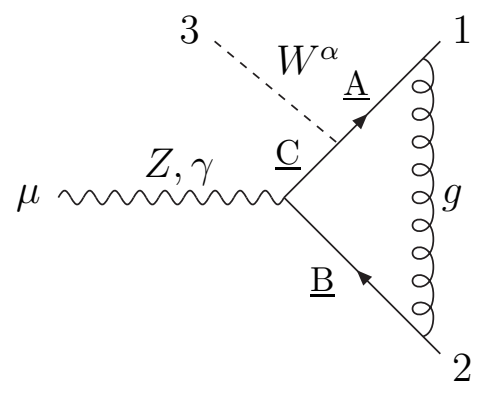

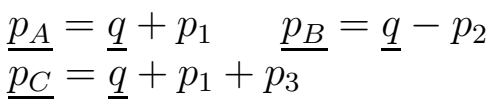

$$
\begin{aligned}
& D_{1}=\underline{q}^{2} \\
& D_{2}=\left(\underline{q}+p_{1}\right)^{2} \\
& D_{3}=\left(\underline{q}+p_{1}+p_{3}\right)^{2} \\
& D_{4}=\left(\underline{q}-p_{2}\right)^{2}
\end{aligned}
$$

Figure 5: QCD one-loop box diagram. 
The spinorial structure reads

$$
\begin{aligned}
\mathcal{A}^{\alpha \mu} & =-\{\underline{\beta} \underline{A} \alpha \underline{C} \mu \underline{B} \underline{\beta}\}_{12}^{+-} \\
& =2\{B \mu C \alpha A\}_{12}^{+-}+\epsilon\{q \alpha C \mu q\}_{12}^{+-} \\
& -2 \tilde{q}^{2}\left[\{\mu \alpha A\}_{12}^{+-}+\{B \mu \alpha\}_{12}^{+-}+\{\mu C \alpha\}_{12}^{+-}\right] \\
& -\epsilon \tilde{q}^{2}\left[\{\alpha \mu q\}_{12}^{+-}+\{q \alpha \mu\}_{12}^{+-}+\{\alpha C \mu\}_{12}^{+-}\right] .
\end{aligned}
$$

Decomposing the integration as in eq. (44), one gets

$$
\int d^{n} q \frac{\tilde{q}^{2}}{D_{1} D_{2} D_{3} D_{4}}=\int d^{n} q \frac{\tilde{q}^{2} q^{\beta}}{D_{1} D_{2} D_{3} D_{4}}=0+\mathcal{O}(\epsilon) .
$$

In addition

$$
\int d^{n} q \frac{\{q \alpha C \mu q\}_{12}^{+-}}{D_{1} D_{2} D_{3} D_{4}}
$$

does not contain any pole. In fact, infrared divergences are absent by power counting and, in the kinematical regions where collinear divergences take place - namely when $q \propto p_{1}$ or $q \propto p_{2}$ - the numerator of eq. (48) vanishes thanks to the Dirac equation. Therefore, in the limit $\epsilon \rightarrow 0$, only the first term in eq. (46) survives

$$
\mathcal{A}^{\alpha \mu}=2\{B \mu C \alpha A\}_{12}^{+-} .
$$

Notice that, since the diagram is free from ultraviolet divergences, this result coincides with what one would get by assuming eq. (35), as expected.

Terms containing up to three powers of $q$ appear in eq. (49). One could in principle extract the $q$ dependence by means of eq. (20), but this would cause the appearance of $\left(p_{1} \cdot p_{2}\right)$ into the denominator. This can be avoided as follows. Starting, for example, from

$$
2\{q \mu q \alpha 1\}_{12}^{+-}
$$

one gets, after two anticommutations

$$
2\{q \mu q \alpha 1\}_{12}^{+-}=4\{\alpha\}_{12}^{+-}\left[q^{2} p_{1}^{\mu}-2\left(q \cdot p_{1}\right) q^{\mu}\right]+4 p_{1}^{\alpha}\{q \mu q\}_{12}^{+-}
$$


and, rewriting the last term as a trace

$$
\begin{aligned}
\{q \mu q\}_{12}^{+-} & =\frac{1}{N} \operatorname{Tr}\left[q 231 q \mu \Pi_{+}\right]=\frac{1}{N}\left[2\left(q \cdot p_{2}\right)\{q \mu 3\}_{11}^{+-}\right. \\
& \left.-2\left(q \cdot p_{3}\right)\{q \mu 2\}_{11}^{+-}+2\left(q \cdot p_{1}\right)\{\mu q 3\}_{22}^{+-}-q^{2}\{32 \mu\}_{11}^{+-}\right] \\
N & =\{3\}_{21}^{+-},
\end{aligned}
$$

from which the reconstruction of denominators can be performed as usual. In eq. (52), $N$ appears into the denominator, instead of $\left(p_{1} \cdot p_{2}\right) . N$ does not vanish in the collinear limit $p_{1} \propto p_{2}$, but gives zero when $p_{3} \propto p_{1}$ or $p_{3} \propto p_{2}$. However, since $p_{3}$ refers to a massive particle, those configurations practically never occur in numerical programs, so that the described reduction gives numerically stable expressions.

All terms in eq. (49) can be reduced as shown. The complete result is presented in appendix A. Notice that the reduction can be worked out exactly in the same way even when one of the internal fermion lines of the diagram is massive. Only denominators change. For example, with the replacement $\left(p_{1}+p_{3}\right)^{2} \rightarrow\left(p_{1}+p_{3}\right)^{2}-m_{t}^{2}$, the result in appendix $\mathrm{A}$ can also be used to compute the box diagram that one encounters in evaluating QCD corrections to off-shell single top production at hadron colliders [20].

As a last example, in order to introduce the algorithm in the presence of external massless vector particles, I show the reduction of the QCD fourpoint function in fig. 6, appearing in the calculation of the $W$ decay into 3 jets.

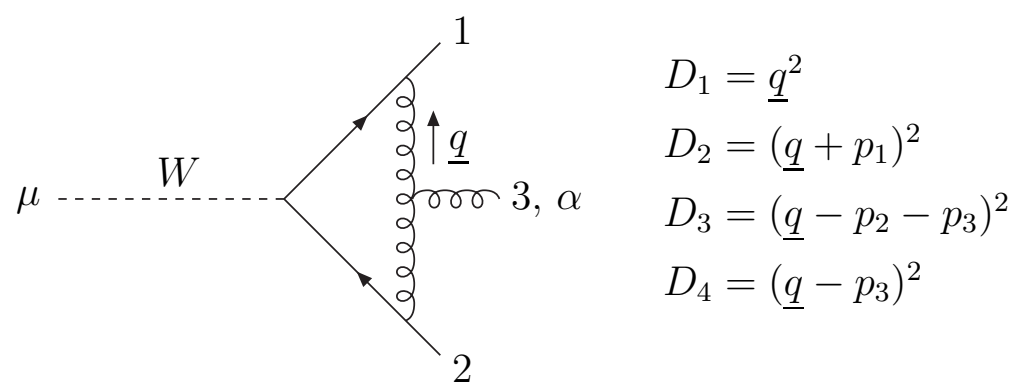

Figure 6: Box diagram in $W \rightarrow 3$ jets. 
As before, all terms in $\epsilon$ dimensions do not contribute, when the limit $\epsilon \rightarrow 0$ is taken, so that only four-dimensional objects appear in the numerator of the diagram

$$
\begin{aligned}
\mathcal{A}^{\mu}(\lambda) & =\left\{\beta\left(q+p_{1}\right) \mu\left(q-p_{2}-p_{3}\right) \delta\right\}_{12}^{+-} V^{\beta \alpha \delta} \epsilon_{\alpha}\left(\lambda, p_{3}\right) \\
V^{\beta \alpha \delta} & =g^{\beta \alpha}\left(-q-p_{3}\right)^{\delta}+g^{\alpha \delta}\left(2 p_{3}-q\right)^{\beta}+g^{\delta \beta}\left(2 q-p_{3}\right)^{\alpha} .
\end{aligned}
$$

The polarization vector of the gluon can be expressed in terms of spinors by means of eq. (6). For example, by choosing $b=p_{1}$ when $\lambda=-1$, one gets

$$
\begin{aligned}
\epsilon_{\alpha}\left(-1, p_{3}\right) & =\frac{1}{N}\{\alpha\}_{31}^{+-} \\
N & =\sqrt{2}<13>^{*} .
\end{aligned}
$$

Therefore, eq. (53) can be rewritten in terms of single traces

$$
\begin{aligned}
\mathcal{A}^{\mu}(-1) & =\frac{2}{N}\left[\left\{p_{1} q \mu\left(q-p_{2}-p_{3}\right)\left(q+p_{3}\right)\right\}_{32}^{+-}\right. \\
& +\left\{\left(q-p_{2}\right) \mu\left(q+p_{1}\right)\left(q-2 p_{3}\right) p_{1}\right\}_{32}^{+-} \\
& \left.-2\left\{q p_{1}\left(q-p_{2}-p_{3}\right) \mu\left(q+p_{1}\right)\right\}_{32}^{+-}\right]
\end{aligned}
$$

and the reconstruction of denominators performed in the usual way. For completeness, I list the result in appendix A.

\section{Conclusions}

I have presented a method to simplify the tensorial decomposition of loop diagrams in $n$ dimensions. Rank $l$ tensor integrals, with at least two massless momenta, can be reduced to at most rank one tensor functions with the initial number of denominators, plus integrals with less denominators and rank $<l$. A better control of the numerical instabilities due to the appearance of Gram determinants is possible, with respect to traditional reduction techniques.

For processes where all heavy particles decay, the class of the most complicated loop diagrams lies in the domain of validity of the proposed method, allowing strong simplifications.

To illustrate the approach, I computed diagrams contributing to several processes of physical interest. 


\section{Acknowledgment}

I wish to thank G. Passarino for stimulating and useful discussions and for reading the manuscript.

\section{Appendix A}

In this appendix I present the complete reduction of the diagrams in fig. 5 and 6 .

The amplitude of the diagram in fig. 5 is

$$
\mathcal{M}^{\alpha \mu}=\int d^{n} q \frac{\mathcal{A}^{\alpha \mu}}{D_{1} D_{2} D_{3} D_{4}}
$$

with $\mathcal{A}^{\alpha \mu}$ given in eq. (49).

One gets, in terms of denominators and $N=\{3\}_{21}^{+-}$, the following expression

$$
\begin{aligned}
& \mathcal{A}^{\alpha \mu}=2\left[\{q \mu(1+3) \alpha 1\}_{12}^{+-}-\{2 \mu(q+1+3) \alpha 1\}_{12}^{+-}-\{2 \mu(1+3) \alpha q\}_{12}^{+-}\right] \\
& +\frac{2}{N}\left\{( D _ { 2 } - D _ { 1 } ) \left[\{\mu q 3 \alpha 1\}_{22}^{+-}-\{q \mu 31 \alpha\}_{22}^{+-}-\{\alpha q 32 \mu\}_{2}^{+-}\right.\right. \\
& +\{\alpha(q+1+3) \mu q 3\}_{22}^{+-}+\frac{\left(p_{1}+p_{3}\right)^{2}}{N}\left(\{\alpha 23 q \mu\}_{21}^{+-}+\{\alpha 23 \mu q\}_{21}^{+-}\right. \\
& \left.\left.-\{\mu q 32 \alpha\}_{21}^{+-}\right)\right]+\left(D_{1}-D_{4}\right)\left[\{q \mu 31 \alpha\}_{11}^{+-}+\{\alpha q \mu 23\}_{11}^{+-}-\{q \alpha 2 \mu 3\}_{1}^{+-}\right. \\
& \left.+\{q \mu(q+1+3) \alpha 3\}_{11}^{+-}-\frac{\left(p_{1}+p_{3}\right)^{2}}{N}\{\alpha 1 q \mu 3\}_{21}^{+-}\right] \\
& +D_{1}\left[\{\mu(1-2) \alpha 23\}_{11}^{+-}-\{32 \mu(1-2) \alpha\}_{11}^{+-}-\{32 \alpha(q+1+3) \mu\}_{11}^{+-}\right. \\
& \left.-\frac{\left(p_{1}+p_{3}\right)^{2}}{N}\left(\{\alpha 231 \mu\}_{21}^{+-}-\{\alpha 132 \mu\}_{21}^{+-}\right)\right]+\left(D_{3}-D_{2}\right)\left[\{q \alpha 2 \mu 2\}_{11}^{+-}\right. \\
& \left.-\{q \mu 21 \alpha\}_{11}^{+-}-\{q \mu(q+1+3) \alpha 2\}_{11}^{+-}+\frac{\left(p_{1}+p_{3}\right)^{2}}{N}\{\alpha 1 q \mu 2\}_{2}^{+-}\right] \\
& +\left(p_{1}+p_{3}\right)^{2}\left[\{q \mu 21 \alpha\}_{11}^{+-}-\{q \alpha 2 \mu 2\}_{11}^{+-}+\{q \mu(1+3) \alpha 2\}_{11}^{+-}\right.
\end{aligned}
$$




$$
\left.\left.-\frac{\left(p_{1}+p_{3}\right)^{2}}{N}\{\alpha 1 q \mu 2\}_{2}^{+-}\right]\right\} .
$$

The above equation has been numerically checked against the result in ref. [19], that was obtained using the standard reduction techniques of ref. [1].

As for the diagram in fig. 6 (with gluon polarization $\lambda=-1$ ), one has

$$
\mathcal{M}^{\mu}(-1)=\int d^{n} q \frac{\mathcal{A}^{\mu}(-1)}{D_{1} D_{2} D_{3} D_{4}},
$$

with

$$
\begin{aligned}
& \mathcal{A}^{\mu}(-1)=\frac{2}{N}\left\{\left(2 D_{4}-D_{3}\right)\{1 q \mu\}_{32}^{+-}+2 D_{4}\{1 \mu q\}_{32}^{+-}\right. \\
& +\left(D_{2}-4\left(p_{1} \cdot p_{3}\right)\right)\{(q-2) \mu 1\}_{32}^{+-} \\
& +2\left(D_{1}-D_{2}\right)\left[\{q \mu(q+1)\}_{32}^{+-}+\{2 q \mu\}_{32}^{+-}\right] \\
& +2 D_{1}\left[\{1 \mu 1\}_{32}^{+-}-\{12 \mu\}_{32}^{+-}\right]+2\{2 \mu q 31\}_{32}^{+-} \\
& \left.+2\{q 1(2+3) \mu 1\}_{32}^{+-}\right\}, \quad N=\sqrt{2}<13>^{*}
\end{aligned}
$$

\section{References}

[1] G. Passarino and M. Veltman, Nucl. Phys. B160 (1979) 151.

[2] G. J. van Oldenborgh and J. A. M. Vermaseren, Z. Phys. C46 (1990) 425 ;

A. Signer, Helicity method for next-to-leading order corrections in QCD, Ph.D. thesis, ETH Zürich (1995).

[3] A. I. Davydychev, Phys. Lett. B263 (1991) 107;

O. V. Tarasov, Phys. Rev. D54 (1996) 6479.

[4] Z. Bern, L. Dixon and D. A. Kosower, Nucl. Phys. B412 (1994) 751;

Phys. Lett. B302 (1993) 299; Phys. Lett. B318 (1993) 649.

[5] J. M. Campbell, E. W. N. Glover and D. J. Miller, One-loop Tensor Integrals in Dimensional Regularisation, hep-ph/9612413. 
[6] Z. Bern and A. G. Morgan, Nucl. Phys. B467 (1996) 479;

Z. Bern, L. Dixon and D. A. Kosower, Progress in one-loop QCD computations, hep-ph/9602280.

[7] Z. Kunszt, A. Signer and Z. Trócsányi, Phys. Lett. B336 (1994) 529;

A. Signer, Phys. Lett. B357 (1995) 204.

[8] F. A. Berends et al., Phys. Lett. 103B (1981) 124;

P. De Causmaecker at al., Nucl. Phys. B206 (1982) 53;

R. Kleiss and W. J. Stirling, Nucl. Phys. B262 (1985) 235;

J. F. Gunion and Z. Kunszt, Phys. Lett. 161B (1985) 333;

Z. Xu, D. -H. Zhang and L. Chang, Nucl. Phys. B291 (1987) 392.

[9] G. Passarino, Phys. Rev. D28 (1983) 2867;

R. Pittau, The $e^{+} e^{-}$annihilation processes with final fermionic states as a test of the Standard Model of the electroweak interactions, Ph.D. thesis, DFTT 11/93, March 1993.

[10] J. G. M. Kuijf, Multiparton production at hadron colliders, Ph.D. thesis, 1991

F. A. Berends and W. T. Giele, Nucl. Phys. B294, 700 (1987).

[11] T. Brodkorb, J. G. Körner and E. Mirkes, Phys. Lett. B216 (1989) 203.

[12] J. Kahane, J. Math. Phys. 9 (1968) 1732;

J. S. R. Chisholm, Comput. Phys. Commun. 4 (1972) 205;

E. R. Caianiello and S. Fubini, Nuovo Cimento 9 (1952) 1218.

[13] T. Lee, Phys. Rev. D55 (1997) 2591.

[14] M. Veltman, Nucl. Phys. B319 (1989) 253.

[15] Z. Bern and D. A. Kosower, Nucl. Phys. B379 (1992) 451.

[16] D. Kreimer, Phys. Lett. B237 (1990) 59;

J. G. Körner, D. Kreimer and K. Schilcher, Z. Phys. C54 (1992) 503.

[17] R. Mertig and W. L. van Neerven, Z. Phys. C70 (1996) 637.

[18] G. D. Mahlon, Phys. Rev. D49 (1994) 2197 and Phys. Rev. D49 (1994) 4438. 
[19] E. Maina, R. Pittau and M. Pizzio, Phys. Lett. B393 (1997) 445.

[20] R. Pittau, Phys. Lett. B386 (1996) 397. 